better anti-HIV drugs.

The protein CCR5 resides on the surface of certain immune cells. A team led by Beili Wu at the Chinese Academy of Sciences' Shanghai Institute of Materia Medica successfully crystallized CCR 5 bound to the anti-HIV drug maraviroc. The structure suggests that the drug works by making CCR 5 more rigid, and so prevents movements that allow HIV to enter cells. It also reveals why such HIV drugs fail to work with certain CCR 5 mutants.

Differences between CCR 5 and CXCR4, another protein used by HIV to enter cells, may explain why some HIV strains favour one protein gateway over the other.

Science http://doi.org/ntj (2013) ZOOLOGY

\section{Fertility smells like preen spirit}

A bird's scent may indicate how many offspring it will produce.

Danielle Whittaker of Michigan State University in East Lansing and her colleagues analysed compounds that evaporate from the oily secretions that birds spread over their feathers when preening. The team collected oil from 12 female and 22 male dark-eyed juncos (Junco hyemalis; pictured) and found that the oil's chemical profile differed between the sexes.

Birds that released more chemicals characteristic of their sex produced more offspring. And males with more 'female' odours fledged more hatchlings fathered by other birds from their nests. Overall, the smell of a bird was a better predictor of reproductive success than either size or plumage. Anim. Behav. http:// doi.org/nr3 (2013)

\section{African tree gets to the root of pain}

An African plant used in traditional medicine for pain relief contains the same active ingredient as an artificial pain killer.

Together with scientists in Cameroon, France and Switzerland, Michel De Waard at Joseph Fourier University in Grenoble, France, collected extracts from the pincushion tree (Nauclea latifolia), separated compounds into groups on the basis of their mass and chemical properties, then tested each group in mice for its ability to relieve pain.

The team found an oily yellow compound in the most potent group and determined its chemical structure to be that of tramadol, which has been sold as a synthetic analgesic since the 1970s. The compound was detected only in the plant's roots - the same parts used in traditional remedies to treat pain. The researchers believe that this is the first time a widely prescribed synthetic drug has been found in a plant at clinically relevant concentrations.

Angew. Chem. http://doi.org/ f2dv27 (2013)

\section{Ancient bear bone yields a sequence}

The mitochondrial genome of a Pleistocene cave bear (Ursus deningeri) has been reconstructed using extremely short DNA molecules from a bone that is more than 300,000 years old. Apart from rare specimens preserved in permafrost, the fossil is some 200,000 years older than any other material used to generate a complete DNA sequence. By reworking methods to purify the tiny amounts of damaged DNA that are typical of old samples,

COMMUNITY CHOICE

The most viewed papers in science

\title{
Fruits shrink diabetes risk
}

\section{HIGHLY READ \\ on www.bmj.com in August}

Three large, long-running health surveys suggest that some fruits - but not fruit juice - reduce the risk of diabetes.

Researchers led by Qi Sun at the Harvard

School of Public Health in Boston, Massachusetts, looked at people's reports of how often they ate certain fruits and whether they developed diabetes. Results came from a total of 187,382 US health professionals whose health and habits have been tracked for more than two decades, during which time more than 12,000 of the study participants developed diabetes.

Eating whole fruits such as apples, pears, grapes, raisins and particularly blueberries was associated with a lower risk of diabetes. By contrast, drinking fruit juice was associated with a slight increase in risk, perhaps because juices contain fewer fruit-specific chemicals and introduce more sugar into the bloodstream more quickly.

Br. Med. J. 347, f5001 (2013)

Jesse Dabney at the Max Planck Institute for Evolutionary Anthropology in Leipzig, Germany, and his colleagues collected and sequenced DNA strands as short as 30 basepairs. More than $90 \%$ of the sequences used to produce the genome were less than 50 basepairs long - too short to be used efficiently with conventional methods.

The technique could allow researchers to recover DNA from ancient humans and other specimens that are currently considered too degraded for most analyses. Proc. Natl Acad. Sci. USA http://doi.org/nr4 (2013)

\section{VOLCANOLOGY}

\section{Eruption sent microbes flying}

Fragments of once-living creatures inside volcanic rocks can help to trace an eruption to its source.

New Zealand's North Island is blanketed in debris from a super-eruption of the Taupo volcano at the island's centre, which happened some 25,000 years ago. A team led by Alexa Van Eaton at the Victoria University

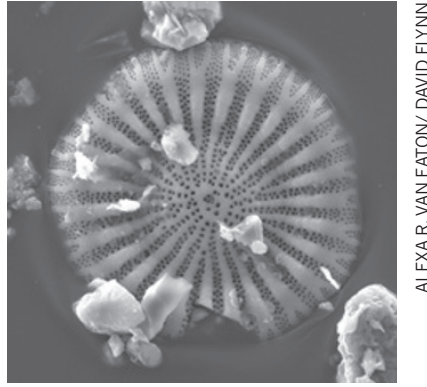

of Wellington searched for microfossils in rocks as far as 850 kilometres from the volcano. They found abundant skeletons of algae known as diatoms (pictured), including a type that lives only in lakes on the North Island. This confirms the findings of earlier work that the eruption blasted through a lake on that island.

Such fossils could help volcanologists to work out the locations and environmental settings of past eruptions, the authors say. They speculate that volcanoes might even disperse living cells across long distances.

\section{Geology http://doi.org/nr8} (2013)

\section{DNATURE.COM}

For the latest research published by Naturevisit:

www.nature,com/latestresearch 